DOI 10.1007/s10553-021-01250-x

Chemistry and Technology of Fuels and Oils, Vol. 57, No.2, May, 2021 (Russian Original No.2, March - April, 2021)

\title{
FORECASTING OIL DEMAND WITH THE DEVELOPMENT OF COMPREHENSIVE TOURISM
}

\author{
Yanrong Huang ${ }^{1}$, Shuaihao Li $^{2,3}$, Rui Wang ${ }^{4,5}$, Zhijiang Zhao ${ }^{1}$, Bin Huang ${ }^{1}$, \\ Bo Wei ${ }^{6}$, Guangming $\mathrm{Zhu}^{1}$
}

The prediction of oil demand is an important issue related to national energy security and economic development. With the COVID-19 outbreak, the international oil price fluctuates sharply, and oil consumption growth slows down. Therefore, accurate prediction of oil demand plays an important practical and theoretical role. In this paper, in accordance with the Chinese state policy of stimulation of domestic demand for energy resources, we have selected 15 major factors and analyzed their influence on the domestic oil demand from the perspective of comprehensive tourism analysis. Based on the data analysis of oil consumption from 2000 to 2018, four neural network methods are used to predict the influence of selected factors on oil consumption demand in China. The experimental results show that the best correlation is obtained between domestic tourism revenue and total tourism expenditure factors and oil demand, and the Layer Recurrent Neural Network method has high prediction accuracy, stronger stability, and the best performance.

Keywords: oil demand, forecasting, comprehensive tourism, recurrent neural network.

${ }^{1}$ College of Economics \& Management, Zhejiang University of Water Resource and Electric Power, Hangzhou 310018, China. ${ }^{2}$ School of International Business and Management, Sichuan International Studies University, Chongqing, 400031, China. ${ }^{3}$ Research Center for International Business and Economy, Sichuan International Studies University, Chongqing, 400031, China. ${ }^{4}$ School of Economics \& Management, Jiangxi University of Science and Technology, Ganzhou 341000, China. ${ }^{5}$ School of Economics, Fujian Agriculture and Forestry University, Fuzhou 350000, China. ${ }^{6}$ School of Informatics Science and Technology, Zhejiang Sci-Tech University, Hangzhou 310018, China. Corresponding author: Yanrong Huang.E-mail:hyanrong@whu.edu.cn. Translated from Khimiya i Tekhnologiya Toplivi Masel, No. 2, pp. 61 - 66, March - April, 2021. 


\section{INTRODUCTION}

Oil plays an important role in ensuring national energy security and economic development. The petroleum industry is one of the basic strategic industries of the national economy and the people's livelihood and the driver of the national economy, ensuring national energy security and the smooth operation of the industrial chain. At present, China is cooperating with the international COVID-19 strategies, the oil price is increasing sharply and oscillating, and the global economic recession is stacked. The Chinese government has been pushing the domestic demand under the background of the Chinese government's implementation. Therefore, the prediction of oil demand is of practical significance and theoretical value.

According to the data of the China Statistical Yearbook, the oil consumption in 2018 was 622.45 million tons, including 227.39 million tons of transport fuel consumption, 73.28 million tons of domestic consumption, and 224.6 million tons of industrial oil consumption. Fuel is the principal component of terminal oil consumption. According to the historical statistical data of most developed countries and regions in the world for more than 100 years, primary energy consumption [1,2], the terminal energy consumption of the industrial sector [3], and terminal energy consumption of the transportation sector are related to the level of economic development. The transportation sector dominates more than $65 \%$ of oil consumption both in developed countries such as the United States, the United Kingdom, and Germany, and developing countries such as China and India [4]. Experience shows that the number of motor vehicles has a crucial impact on the transportation sector's oil consumption. Since 2000, China's passenger car market has experienced explosive growth. In 2020, China's vehicle ownership reached 372 million, and it is expected that China's vehicle ownership will be the highest in the world in 2021 [5]. The rapid increase in the number of passenger cars drives the consumption of gasoline.

At present, with the development of China's economy, improvement of people's living standards, domestic tourism popularization, and normalization of tourism consumption, tourism has become one of the most important lifestyles. Comprehensive tourism is a new concept put forward by Li Jianxin and other scholars in 2013. It is believed that comprehensive tourism is a new tourism mode that integrates multiple industries and multiple subjects, promotes the development of tourism destinations from the perspective of all factors, all industries, all processes, all directions, all periods and destinations, and provides a more satisfactory travel experience [6]. J. F. Lv believed that comprehensive tourism needs to integrate various types of regional tourism resources, enrich tourism products, and promote the development of spatial panoramic and industrial integration [7]. J. Li pointed out that "comprehensive tourism" is a new model of regional coordinated development through the organic integration of regional tourism resources, public service resources, and other economic and social resources [8]. Wang et al. believed that the key to comprehensive tourism strategy implementation is to intrinsically integrate all kinds of tourism-related resources in the region [9]. At present, scholars analyze the comprehensive tourism model from the perspectives of "system theory," "resource integration," and "industrial integration."

\section{LITERATURE REVIEW}

At present, many studies have been focused on energy demand forecasting at home and abroad. From the forecast data, Günay applied the artificial neural network model of time series to forecast the annual power demand in Turkey, which involved data on population, per capita GDP, inflation rate, and relevant parameters of power consumption from 1975 to 2013 [10]. Chen et al. used the leap model to predict the energy demand of Changsha City in Hunan Province from 2015 to 2020 under different scenarios and discussed the impact of GDP growth, industrial structure, and energy-saving goals on future energy demand [11]. Haldenbilen et al. applied the genetic algorithm including GDP, population, and vehicle driving distance to predict the energy demand of 
the transportation sector [12]. Yu et al. proposed an online big data-driven oil consumption prediction model based on Google Trends [13]. Ji used the advanced direct search algorithm method to optimize the model parameters and applied an improved logistic model to describe and forecast China's oil consumption data [14]. From the perspective of forecasting methods, there are two kinds of econometric and machine learning methods, such as time series analysis method, strategic planning method, grey prediction model, input-output analysis method, regression analysis method, artificial neural network, trend recurrence method, etc. Wang et al. constructed a grey prediction model GMC based on convolution integral to predict China's industrial sector energy. The results show that the prediction GMC model is more accurate than the traditional Sarma and GM methods [15]. Li et al. used a neural network combination model to forecast China's oil demand and put forward that the TCM-NNCT neural network is a feasible and effective method to forecast China's oil demand [16]. Li et al. used the factor analysis method and the logistic model to establish a prediction model of oil consumption demand based on scenario analysis and carried out verification and error analysis on the model [17]. Wen et al. selected three prediction models: grey system model, cubic exponential smoothing model, and BP neural network model. The research results show that the neural network method can achieve a good prediction accuracy [18]. To sum up, oil consumption is closely related to economic development [18], which is affected by social and economic factors including population, economic structure, consumption structure, number of vehicles (motor vehicles), petrochemical industry, energy conservation, oil substitution, and scientific and technological progress $[19,20]$. Most of the existing methods of oil demand forecasting are based on macro factors such as per capita GDP, GDP growth rate, and industrial indexes. They do not reflect the inherent characteristics and trends of residents' living standards and energy consumption in the forecasting model from the perspective of micro individuals and residents. The research results show that the use of the neural network method is an effective method of oil demand forecasting [21], but the potential of using different neural network models to improve the accuracy of oil demand forecasting has not been discussed by scholars. From the perspective of comprehensive tourism, this paper systematically considers the factors that can affect the oil consumption demand such as population, residents' consumption level, car ownership, and domestic and foreign travel, analyzes the importance of the influencing factors, and uses a variety of neural network models to predict and discuss oil consumption demand.

\section{RESEARCH METHODS}

\subsection{SELECTION METHOD OF INFLUENCING FACTORS}

The oil consumption demand is affected by many factors. Combined with the research results in the field of oil demand forecast, the paper analyzes China's oil consumption over the years from 2000 to 2018 , following the principles of comprehensiveness, comparability, and availability. The expert scoring method is used to determine the oil demand influencing factors., The main influencing factors of oil consumption demand are summarized and screened from the perspective of comprehensive tourism.

\subsection{GREY CORRELATION ANALYSIS METHOD}

Grey correlation analysis can measure the correlation degree between the system factors according to the development and change trend of those factors. The calculation steps are as follows:

Suppose that the original data of oil consumption in the $n^{\text {th }}$ year are dimensionless processed as $X_{0}(n)$; then a reference data column is generated after dimensionless processing of $n$-year oil consumption, as shown by the formula 


$$
X_{0}=\left[X_{0}(1), X_{0}(2) \ldots X_{0}(n)\right]
$$

The index $i$ refers to a factor affecting oil consumption demand, where $m$ is the number of factors considered, and $i \in(1,2, \cdots, m)$. The comparison data set is generated after dimensionless processing, as expressed by

$$
X_{i}=\left[X_{i}(1), X_{i}(2) \ldots X_{i}(n)\right], i \in(1,2, \ldots, m)
$$

For $X_{i}$, the correlation coefficient at point $K$ is expressed as $\xi_{i}(K)$ :

$$
\xi_{i}(K)=\frac{\min _{i} \min _{K}\left|X_{0}(K)-X_{i}(K)\right|+\operatorname{\rho max}_{i} \max _{K}\left|X_{0}(K)-X_{i}(K)\right|}{\left|X_{0}(K)-X_{i}(K)\right|+\operatorname{\rho max}_{i} \max _{K}\left|X_{0}(K)-X_{i}(K)\right|}
$$

Among $\rho \in[0,1]$ usually $\rho=0.5$. Then the correlation degree $r_{i}$ between the influencing factors of oil demand can be expressed as follows:

$$
r_{i}=\frac{1}{n} \sum_{K=1}^{n} \xi_{i}(K)
$$

The higher the value of the correlation degree, the greater the correlation between the comparison sequence and the reference sequence.

\subsection{NEURAL NETWORK PREDICTION METHOD}

Back Propagation Neural Network (BPNN) is a kind of multilayer feed-forward neural network [22]. A BP neural network usually has three or more layers, including the input layer, middle layer (hidden layer), and output layer. The network is fully connected if each cell provides input to every cell at the next level. Each output unit accepts the weighted sum of the output of the previous unit as the input. Through different levels of input and output mapping, it can deal with complex internal logic structure. To achieve the effect of adaptive learning, BP neural network adjusts the weights of neurons through training and learning rules. In this paper, a feed-forward BackProp and a cascade forward neural networks are used for comparative analysis.

The Elman Neural Network was put forward by J. L. Elman in 1990. The Layer Recurrent Neural Network is one of the deep learning algorithms, which is composed of the input layer, the hidden layer with a feedback unit, and the output layer. To improve the approximation ability of unknown nonlinear smooth function, the recurrent neural network introduces internal feedback internal state information into the hidden layer.

Assuming that the learning data input in the time series is expressed as $X=\left\{X_{1}, X_{2}, \ldots, X_{\tau}\right\}$, the expanded length of the Recurrent Neural Network is $\tau$, the evolution direction of time series is called the time step, the assumed time step is $t, h$ is the system status of the RNN, and $s$ is internal status, where $s=s(h, X, y)$.

Because the internal state of the previous time step is needed to solve the current system state, the calculation of loop unit includes recursion. Let $f$ be an excitation function or an encapsulated feed-forward neural network and $\theta$ be a weight coefficient in the cycle unit, which is independent of the time step; then the cycle unit of the RNN can be expressed as 


$$
h^{(t)}=f\left(s^{(t-1)}, X^{(t)}, \theta\right)
$$

The network structure of the loop formed by the RNN enables neurons to receive information from other neurons as well as their own, which is widely used in many fields.

\subsection{DATA PREPROCESSING METHOD}

The original data cannot be directly used for model construction but must be pre-processed to improve the quality of data mining. The data pre-processing methods in this paper include missing value processing and data standardization processing. The expectation maximization (EM) method is used in this paper to fill in the missing data of residents' car ownership in 2001 and 2002.

In this paper, data standardization is used to process the data, and the conversion function $s$ follows:

$$
x^{*}=\frac{x-x_{\min }}{x_{\max }-x_{\min }}
$$

where $x^{*}$ is the averaged data of $x, x_{\min }$ is the minimum value of $x$, and $x_{\max }$ is the maximum value of $x$.

\subsection{EVALUATION STANDARD OF PREDICTION METHOD}

Mean Absolute Error (MAE), Root Mean Square Error (RMSE), and Mean Absolute Percentage Error $(M A P E)$ methods were used to evaluate the prediction accuracy of the four prediction models, and the feasibility and effectiveness of different neural network prediction methods were verified.

Suppose $y_{i}$ is the predicted value of oil demand in year $i$, where $i \in(1,2, \ldots, n), \hat{y}_{i}$ is the actual consumption of oil, and $n$ is the total number of samples. The MAE method is used to describe the difference between the observed value and the fitted value and to reflect the discrete degree of each observation value of the sample, as expressed by the following formula:

$$
\begin{array}{r}
A E=\left|y_{i}-\hat{y}_{i}\right| \\
M A E=\frac{1}{n} \sum_{i=1}^{n}\left|y_{i}-\hat{y}_{i}\right|
\end{array}
$$

$R M S E$ is a measure of accuracy and reliability; the expression is as follows:

$$
R M S E=\sqrt{\frac{\sum_{i=1}^{n}\left(y_{i}-\widehat{y}_{i}\right)^{2}}{n}}
$$

$M A P E$ can accurately reflect the actual prediction error and eliminate the problem of mutual cancellation; the expression is

$$
M A P E=\frac{1}{n} \sum_{i=1}^{n}\left|\frac{y_{i}-\hat{y}_{i}}{y_{i}}\right| \times 100 \%
$$




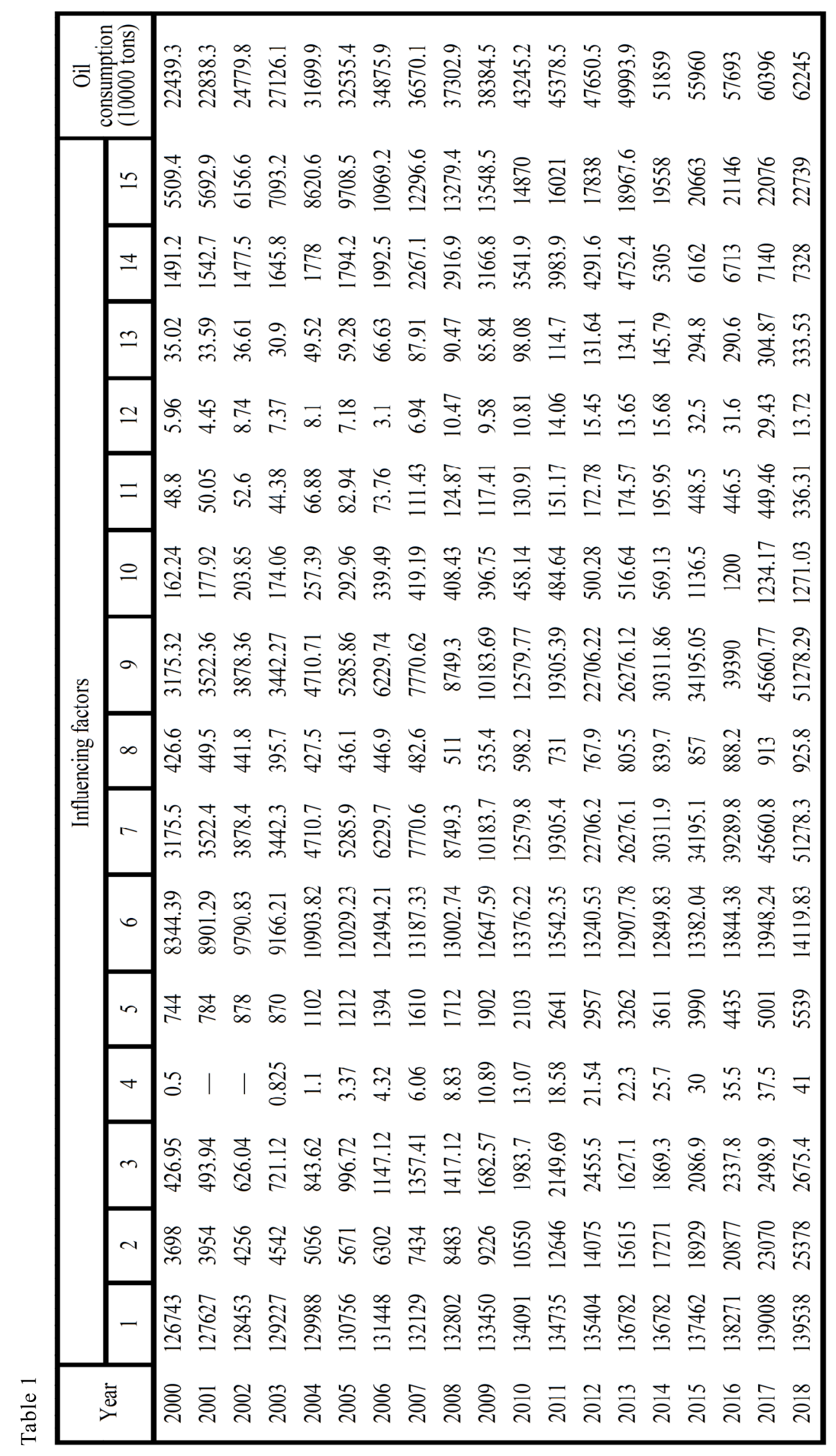




\section{EMPIRICALANALYSIS}

\subsection{DATA SOURCES AND SAMPLE DATA}

In this paper, the data involved in the oil demand forecast are obtained from the China Statistical book 2000-2020 issued by the National Bureau of Statistics of the People's Republic of China. Based on the perspective of comprehensive tourism, this paper considers 15 influencing factors, from 1 to 15 , as follows: the total population of China $(\times 10000)$; consumption level of residents; residents' consumption of transportation and communication; car ownership of residents (100 households); the number of domestic tourists (million); the number of inbound tourists $(\times 10000)$; total cost of domestic tourism; per capita cost of domestic tourism; domestic tourism revenue ( $\$ 100$ million); international tourism revenue ( $\$ 100$ million); international tourism revenue (long-distance transportation); international tourism (automobile) ( $\$ 100$ million); international tourism (civil aviation) ( $\$ 100$ million); oil consumption of household (10000 tons); and oil consumption in transportation (10000 tons).

\subsection{CORRELATION ANALYSIS OF INFLUENCING FACTORS OF OIL CONSUMPTION}

After dimensionless processing of the data in Table 1, the total oil consumption and 15 influencing factors are analyzed by using SPSSAU software. The analysis results are shown in Table 2.

The results show that among the 15 influencing factors, the correlation degree of the total oil consumption is the highest with domestic tourism income and total tourism expenditure, with the correlation degree of 0.84 , followed by transportation, with the correlation degree of 0.802 . The correlation degree between the total population and the total oil consumption in China is the lowest, which is 0.44 . The results show that increase or decrease of the population has little influence on the oil demand. Among all the influencing factors, except the total population index of China, the correlation degree between other factors and the total oil consumption is higher than 0.68 , which indicates that the indexes summarized and screened in this study have a strong correlation degree with the oil demand and are suitable for prediction of oil consumption demand.

\subsection{TEST AND ANALYSIS OF PREDICTION RESULTS}

\subsubsection{Neural network prediction results}

According to the working principle of the neural network model, using MATLAB r2016b software, 15 influencing factors such as domestic tourism revenue, total tourism cost, and transportation oil consumption are taken as input samples, and the total oil consumption is taken as output sample. Taking the oil Table 2

\begin{tabular}{|c|c|c|c|c|c|c|c|c|c|c|c|c|c|c|c|}
\hline & \multicolumn{15}{|c|}{ Influencing factors } \\
\hline 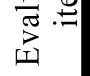 & 1 & 2 & 3 & 4 & 5 & 6 & 7 & 8 & 9 & 10 & 11 & 12 & 13 & 14 & 15 \\
\hline 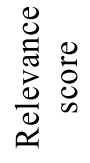 & 0.44 & 0.775 & 0.7 & 0.689 & 0.705 & 0.792 & 0.84 & 0.694 & 0.84 & 0.693 & 0.69 & 0.689 & 0.69 & 0.714 & 0.802 \\
\hline 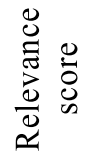 & 15 & 5 & 8 & 13 & 7 & 4 & 2 & 9 & 1 & 10 & 11 & 14 & 12 & 6 & 3 \\
\hline
\end{tabular}


consumption data from 2000 to 2018 , the data set is divided into the training set, verification set, and test set, where $70 \%$ of the samples are used as the training set, $15 \%$ as the verification set, and $15 \%$ as the test set. Four neural network models, Cascade Forward, Feed Forward Backward, Elman Backward, and Layer Recurrent neural network, are used to predict the oil demand.

The accuracy of the test set and verification set differs depending on the hidden layer of the neural network. Therefore, the neuron number of a hidden layer of the neural network is determined to be 10; the input layer is 15 input variables, the output variable is 1 , the output neuron is 3 , the learning rate value is 0.1 , the training accuracy value is 0.00001 , and the maximum training times is 100 . After using MATLAB to train the neural network, the regression results of cascade forward and feed-forward-backward models are shown in Fig. 1.

It can be seen that the target values and output results of the two models are basically fitting on the same line, which shows that the training results are good. Here $R$ is the coefficient of determination, also known as the goodness of fit, which represents the digital characteristics of the relationship between a random variable and multiple random variables. It is a statistical index used to reflect the reliability of the regression model to explain the change of dependent variables. When $R$ is close to 1 , the reference value of the relevant equation is high; on the contrary, when $R$ is close to 0 , the reference value is low. As shown in Fig. 1, the goodness of fit of cascade forward and feed-forward BP neural network models on the training set, verification set, and test set are 0.99656 and 0.99714 respectively, indicating that more than $99.6 \%$ of the oil demand forecast can be explained or determined by the 15 factors selected in this paper.

Four neural network models, Cascade Forward, Feed Forward Backward, Elman Backward, and Layer Recurrent Neural Network, are used to predict the oil demand, and the predicted values of the four neural network models from 2000 to 2018 are obtained. To compare the difference between the predicted value and the target value of different models with higher accuracy, we have taken the Absolute Error $\left(\left|y_{i}-\hat{y}_{i}\right|\right)$ of the difference between the predicted value and the actual value and compared the difference degree between the predicted value and the actual value of different models. As shown in Table 3, the experimental results show that the predicted values of the four prediction methods are close to the actual values, proving that the four models can provide good regression results and achieve satisfactory prediction results.

From the point of accuracy of the four models, there is a relatively large gap between the predicted value and the actual value of the four models in the period of 2000-2004 compared with other years. In 2000, the

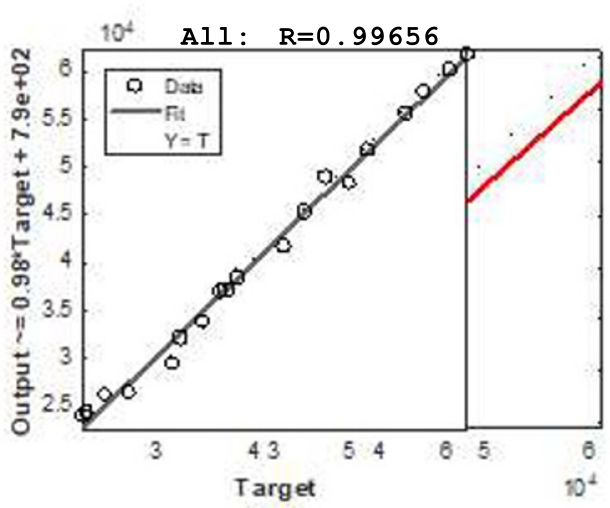

(a)

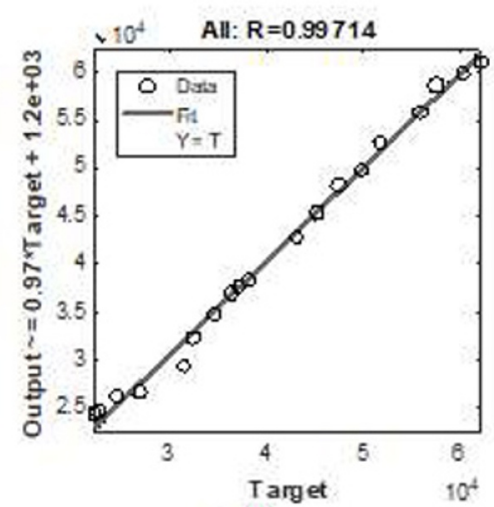

(b)

Fig. 1. R-squared fitting of the prediction model. a) Cascade-Forword Backprop Neural Network; b) Feed-Forward Backprop Neural Network. 


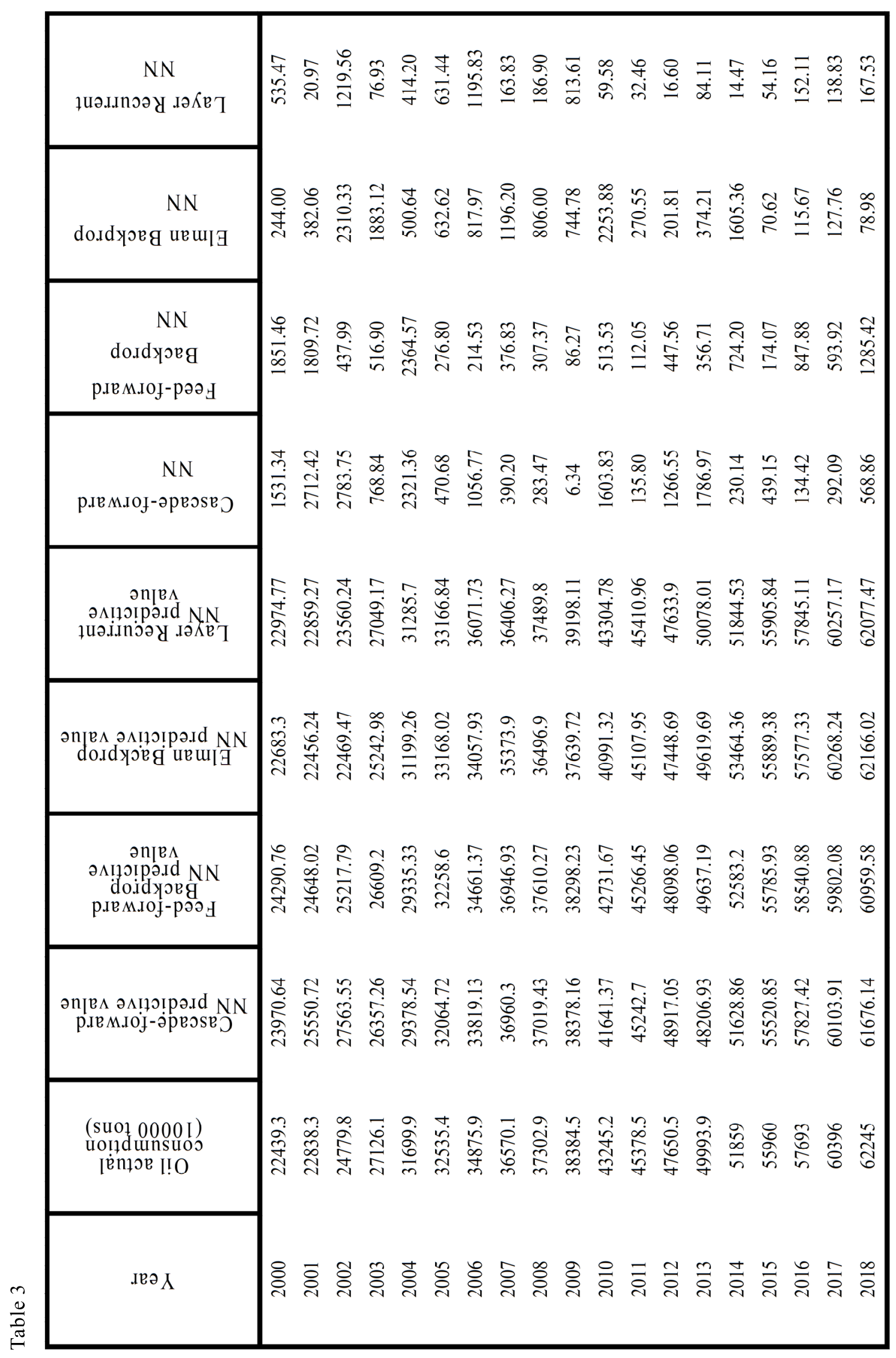


actual oil consumption in China was 224.393 million tons, and the predicted value by Elman BackProp NN model is 239.7064 million tons, which is the closest to the real value, with a difference of 2.44 million tons. The difference between the predicted value and the actual value by cascade forward $\mathrm{NN}$ model is the largest and equals 15.3134 million tons. In 2001, the predicted value of Layer Recurrent Neural Network is the most accurate, the difference between the predicted value and the actual value is only 209700 tons, the ratio of deviation value and actual value, that is, the error rate, is only $0.092 \%$, while the difference between the predicted value and the actual value of cascade forward and feed forward backward is 2712.42 million tons and 1809.72 million tons, respectively, and the deviation is high. From 2010 to 2015, the predicted value of the Layer Recurrent Neural Networks model is closer to the real value than that of the other three models, and the difference from the real value is 59.58 million tons, 32.46 million tons, 16.6 million tons, 84.11 million tons, 14.47 million tons, and 54.16 million tons, respectively, which shows the best prediction performance. The error rate is less than $0.17 \%$, which proves a good prediction accuracy.

Judging from the volatility of the four models and the forecast data from 2000 to 2018 , the Cascade Forward Neural Network model has the maximum deviation of 2783.75 million tons, and the error rate is $11.23 \%$. In 2009 , the predicted value of the model is the closest to the actual value, the difference is 63400 tons, and the error rate is only $0.017 \%$. The deviation between the predicted value and the actual value fluctuates greatly from year to year. The results show that the prediction stability of the Cascade Forward Neural Network model is comparatively poor. The maximum difference between the predicted value and the actual value of the Layer Recurrent Neural Network prediction model in 2006 is 1219.56 million tons, and the maximum difference between the predicted value and the actual value of the Layer Recurrent Neural Network prediction model in 2014 is 14.47 million tons. Among the four models, the fluctuation amplitude of the Layer Recurrent Neural Network prediction model is the smallest, and the prediction stability is better than that of the Cascade Forward Neural Network, Feed-Forward Backward, and Elman Backward prediction models.

\subsubsection{Accuracy test and error analysis}

To test and evaluate the performance of the four models, we have calculated the error values of MAE, RMSE, and MAPE of the four neural network prediction methods, the statistical results are shown in Table 4.

The analysis of MAE, RMSE, and MAPE results in three error estimations. The MAE value is used to describe the difference between the observed value and the fitted value and to reflect the degree of dispersion of the observed values. The MAE values of Cascade Forward, Feed Forward Backward, Elman Backward, and Layer Recurrent Neural Network are 988.58, 699.88, 769.29, and 314.66, respectively. The $M A E$ value of the Layer Recurrent NN model is the smallest, the dispersion degree is the lowest, and the predicted value is the closest to the real value. The RMSE value shows that the predicted value is consistent with the actual value.

Table 4

\begin{tabular}{|l|c|c|c|}
\hline \multicolumn{1}{|c|}{ Methods } & MAE & RMSE & MAPE \\
\hline Cascade-forward NN & 988.58 & 1319.15 & $3.13 \%$ \\
Feed-forward Backprop NN & 699.88 & 945.97 & $2.07 \%$ \\
Elman Backprop NN & 769.29 & 1051.23 & $2.26 \%$ \\
Layer Recurrent NN & 314.66 & 491.51 & $1.02 \%$ \\
\hline
\end{tabular}


The smaller the value, the higher the degree of consistency. Among the four methods, the RMSE value of Cascade Forward NN is the highest, and the Layer Recurrent NN is the lowest. This shows that the gap between the predicted value and the real value of the Cascade Forward NN is the largest, and the predicted value of the Layer Recurrent $\mathrm{NN}$ is the closest to the real value. At the same time, the MAPE average absolute percentage error of the layer recurrent neural network is the lowest. The results show that the MAE, RMSE, and MAPE values of the Layer Recurrent NN are the smallest among the four prediction methods, which indicates that the Layer Recurrent NN model has higher prediction accuracy and stronger performance than the other three models.

\section{CONCLUSIONS}

Oil plays an important role in ensuring national energy security and economic development. Countries are paying more and more attention to the empirical research of oil demand. Demand forecasting methods involve a large number of models and estimation methods. In this paper, we forecast the consumption demand from the perspective of comprehensive tourism analysis. By analyzing and screening the influencing factors of oil consumption, in this paper we have considered the empirical data set of China's oil consumption and influencing factors from 2000 to 2018 and forecasted the oil consumption expectation by the methods of Grey Correlation Analysis, Cascade Forward, Feed Forward Backward, Elman Backward, and Layer Recurrent Neural Network.

From the perspective of comprehensive tourism, this paper puts forward the influencing factors of oil consumption demand and investigates the correlation degree between the selected factors and oil demand through the Grey Correlation Analysis method. The results show that the correlation degree of the oil demand value is the strongest with the domestic tourism income, residents' total tourism cost, and transportation oil consumption, which is $0.84,0.84$, and 0.802 respectively. However, the correlation between China's total population and oil consumption demand is 0.44 , which is the lowest, indicating that the impact of population growth on oil consumption demand is comparatively low.

The statistical data of 15 influencing factors, including the number of domestic tourists, the number of inbound tourists, and the total domestic tourism cost for the period from 2000 to 2018 are obtained from the China Statistical Yearbook. Taking the oil demand influencing factors as the case set, we have used four neural network methods to forecast the oil demand, including the Cascade Forward, Feed Forward Backward, Elman Backward, and Layer Recurrent Neural Network methods. Three error indexes, MAE, RMSE, and MAPE, are applied to test and evaluate the effectiveness of the four neural network methods. The experimental results show that the Layer Recurrent Neural Network prediction method is superior to the other three methods and can provide higher prediction accuracy, stronger stability, and the best performance. In terms of the practical application, the Layer Recurrent NN model has higher prediction accuracy, stronger robustness, and promotion value.

\section{ACKNOWLEDGMENTS}

This work was supported by the Hangzhou Routine Project of Philosophy and Social Science in 2021 under Grant Z21JC098; in part by the Jiangxi Social Science Planning Project under Grant 19GL25; and in part by National College Students' Innovation and Entrepreneurship Training Program.

\section{REFERENCES}

1. A. J. Wang, G. S. Wang, Q. S.Chen, et al., Energy and National Economic Development, Geological Publishing Press, Beijing (2008). 
2. A. J. Wang, G. S. Wang, Q. S.Chen, et al., "S-curve model of relationship between energy consumption and economic development," Nat. Resour. Res., 24, 53-64 (2014).

3. Y. M. Pimenov, A. V. Ulit'ko, and V. A. Sereda, "Method of enhancing the informative content for estimation of the performance properties level of fuels and lubricants," Chem. Technol. Fuels Oils, 56, 186-198 (2020).

4. G. W. Liu, Q. Yan, and J. B. Yang, "World oil demand based on S-curve model of the transport sector," Resour. Sci., 40, 547-557 (2018).

5. Traffic Administration Bureau of the Ministry of Public Security of the People's Republic of China, "In 2020, the number of motor vehicles in China will reach 372 million," Econ. Daily, 1, 1-3 (2021).

6. J. X. Li, L. Y. Zhang, and L. Cui, "Comprehensive tourism: understanding and innovation of building a world class tourism destination - a case study of Beijing," Hum. Geogr., 3, 130-134 (2013).

7. J. F. Lv, "Research on the paradigm of China's comprehensive tourism development from the perspective of urban and rural integration," Henan Sci., 1, 139-142(2014).

8. J. Z. Li, "Great prospects for comprehensive tourism," http://www.cnta.gov.cn/ztwz/zghy/hydt/201508/t20150826_760166.shtml (2015).

9. Y. Y. Wang, "Comprehensive tourism needs new thinking," J. Tourism, 12, 9-11 (2016).

10. M. E. Günay. "Forecasting annual gross electricity demand by artificial neural networks using predicted values of socio-economic indicators and climatic conditions: Case of Turkey," Energy Policy, 90, 92-101 (2016).

11. R. Chen, Z. H. Rao, J..X. Liu, et al., "Prediction of energy demand and policy analysis of Changsha based on LEAP model," Resour. Sci., 39, 482-489 (2017).

12. S. Haldenbilen and H. Ceylan, "Genetic algorithm approach to estimate transport energy demand in Turkey,” Energy Policy, 33, 89-98 (2005).

13. L. Yu, Y. Q. Zhao, L. Tang, et al., "Online big data-driven oil consumption forecasting with Google trends," Int. J. Forecast., 35, 213-223 (2018).

14. L. Q. Ji, “A modified logistic for forecasting petroleum consumption in China," J. China Univ. Pet., 35, 177-181 (2011).

15. Z. X. Wang and P. Hao, "An improved gray multivariable model for predicting industrial energy consumption in China," Math. Model., 40, 5745-5758 (2016).

16. J. R. Li, R. Wang, J. Z.Wang, et al., "Analysis and forecasting of the oil consumption in China based on combination models optimized by artificial intelligence algorithms," Energy, 144, 243-264 (2018).

17. Z. Y. Li, H. Lu, W. P. Ren, et al., "China's oil consumption and its future development trend analysis," Chem. Ind. Eng. Prog., 35(6), 1739-1747 (2016).

18. B. Z. Wen and R. X. Suo, "China's energy demand forecast based on combined model," Math. Pract. Theory, 46(20), 45-53 (2016).

19. N. B. Behmiri and J. R. P. Manso, "The linkage between crude oil consumption and economic growth in Latin America: the panel framework investigations for multiple regions," Energy, 72(7), 233-241 (2014).

20. S. Y. Parka and S. H. Yoo, "The dynamics of oil consumption and economic growth in Malaysia," Energy Policy, 66(3), 218-223 (2014).

21. Y. Yang and J. Wang, "Forecasting wavelet neural hybrid network with financial ensemble empirical mode decomposition and MCID evaluation,” Exp. Syst. Appl., 166,1-18 (2021).

22. D. West, "Neural network credit scoring models," Comput. Oper. Res., 27, 1131-1152 (2000). 